OUCMT-95-6

\title{
Density Fluctuations in Traffic Flow
}

\author{
Satoshi Yukawa ${ }^{1}$ and Macoto KIKUCHI ${ }^{2}$ \\ Department of Physics, Graduate School of Science, Osaka University, \\ Toyonaka 560, Japan
}

\begin{abstract}
Density fluctuations in traffic current are studied by computer simulations using the deterministic coupled map lattice model on a closed single-lane circuit. By calculating a power spectral density of temporal density fluctuations at a local section, we find a power-law behavior, $\sim 1 / f^{1.8}$, on the frequency $f$, in non-congested flow phase. The distribution of the headway distance $h$ also shows the power law like $\sim 1 / h^{3.0}$ at the same time. The power law fluctuations are destroyed by the occurence of the traffic jam.

KEY WORDS: Traffic Flow, Density Fluctuation, $1 / f$ noise, Coupled map lattice
\end{abstract}

The traffic flow presents many interesting phenomena from the viewpoint of physics: one of such phenomena is the $1 / f$ fluctuation in the traffic current [1], 2], which Musha and Higuchi reported for the first time in 1976. They recorded the transit time of the cars on a highway, and calculated its power spectral density (PSD). As a result, they found a power law behavior like $1 / f^{\alpha}$ on the frequency $f$ in low frequency fluctuations and the white noise behavior in high frequency fluctuations. They got the power $\alpha=1$ by fitting the data [1] and got $\alpha=1.4$ by an analysis based on the Burgers equation [2].

Recently, some attempts was made for understanding these power-law fluctuations in the PSD of the traffic current using computer simulations. Takayasu and Takayasu found $1 / f$ fluctuations in the jam phase of their cellular automaton (CA) model by taking into account of the effects of random braking [3]. In the free flow phase, on the other hand, no power law fluctuation was found. In contrast to their results, Nagel and Paczuski reported the $1 / f$ fluctuations near the jamming transition point, using their stochastic CA model[4]. These two studies are based on stochastic CA models on the closed road. Quite recently, another model which describes the two-lane traffic flow was constructed based on a discrete-time continuous-space CA (that is, the coupled map model, in our terminology) by Zhang and $\mathrm{Hu}[5]$; they also found the $1 / f^{\alpha}$ type fluctuations in case of open road with the cars injected from one end.

In this letter, we study the density fluctuations in a traffic flow using the coupled map lattice (CML) model which we proposed recently[6]. In contrast to the CA traffic flow models described above, the present model follows the deterministic dynamics and is simulated on a single-lane circuit.

Here we describe only the essence of the model; details are found in the previous paper[6]. The model is based on the coupled map lattice idea, [7] and thus is constructed in the continuous space and the discrete time. A continuous-space discrete-time model was also introduced by Nagel and Herrmann as the infinitestate version of their CA model. [8]. In the present model, each vehicle is assigned a map which determines the velocity at the next time step using the present values of the headway distance and the velocity as inputs (We define the velocity as the

\footnotetext{
1 e-mail:yuk@phys.sci.osaka-u.ac.jp

2 e-mail:kikuchi@phys.sci.osaka-u.ac.jp
} 
traveling distance during the unit time). We put the map describing a motion of a free vehicle in the following form:

$$
\begin{aligned}
v^{t+1} & =F\left(v^{t} ; v^{F}\right) \\
& \equiv \gamma v^{t}+\beta \tanh \left(\frac{v^{F}-v^{t}}{\delta}\right)+\epsilon
\end{aligned}
$$

where $v^{t}$ is the velocity at time t. $v^{F}$ is the preferred velocity, with which the vehicle tends to run when no other car is found near ahead. $\beta, \gamma, \delta$, and $\epsilon$ are controllable parameters. The free map Eq. 1 describes both (1)the acceleration and the deceleration processes when $v^{t}$ is away from $v^{F}$, and (2)the velocity fluctuation around the preferred velocity when $v^{t}$ is close to $v^{F}$. Throughout this paper, we take $\beta=0.6, \gamma=1.001, \delta=0.1$, and $\epsilon=0.1$. With these parameters, the velocity fluctuation around $v^{F}$ is expressed by a deterministic chaos.

We define interactions between the vehicles by introducing two other maps, which are used according to the headway distance. Both maps determine the next value of the velocity from the present values of the headway distance and the velocity. Suppose the vehicle A has the velocity $v_{A}^{t}$ and is located at the position $x_{A}$ at $t$-th step, and another vehicle B exists ahead of it at the position $x_{B}\left(>x_{A}\right)$. We define the headway distance $h$ as

$$
h=x_{B}-x_{A}+1,
$$

where we take the length of the vehicles as the unit of length. If $h<v_{A}^{t}$, the headway distance is too small to be traveled with $v_{A}^{t}$ so that the vehicle $\mathrm{A}$ is forced to apply sudden braking for avoiding a collision. For expressing the sudden braking process, we employ the similar procedure as what used in ref. [9]; that is, the vehicle A changes its next velocity as $v_{A}^{t+1}=h$. We call this map the sudden braking map. For $v_{A}^{t} \leq h<\alpha v_{A}^{t} \quad(\alpha>1)$, the vehicle changes its next velocity according to the following equation:

$$
\begin{aligned}
v_{A}^{t+1} & =G\left(h, v_{A}^{t} ; v_{A}^{F}\right) \\
& \equiv \frac{F\left(v_{A}^{t} ; v_{A}^{F}\right)-v_{A}^{t}}{(\alpha-1) v_{A}^{t}}\left(h-v_{A}^{t}\right)+v_{A}^{t} .
\end{aligned}
$$

We call it the slowing-down map, which connects the free map and the sudden braking map continuously. When $h>\alpha v_{A}^{t}$, the vehicle A can drive freely and thus the free map Eq. 11 is used. We carry out the simulation using these three maps taking $\alpha=4.0$.

The simulations are made for a single-lane circuit with the number of vehicles fixed. No overtaking is allowed throughout. As the initial state, the positions of the vehicles are randomly chosen and the preferred velocity is uniformly distributed in the range $[2.0,4.0]$. One step of the simulation consists of the following three procedures: first, the headway distance is measured for all vehicles. Next, all the vehicles move simultaneously according to the present velocity. Finally the next velocity is determined from the measured headway distance and the present velocity using the maps introduced above.

We show results of the simulations. The relation of the concentration $\rho$ and the flow $q$ for the whole system, which is called the $\mathrm{q}-\mathrm{k}$ diagram or the fundamental diagram in the traffic engineering, is shown in Fig. 1. This diagram represents the macroscopic properties of the traffic flow. There is a concentration $\rho_{c}$ at which the flow $q$ takes its maximum value [6]. This concentration separates two phases: the free flow phase in lower concentration $\rho<\rho_{c}$, and the congested flow phase in higher concentration $\rho>\rho_{c}$. As we have shown in the previous paper [6], a finite 
spatial region (or regions, in cases of very high concentration) of the traffic jam is formed in the congested flow phase. So we call $\rho_{c}$ as the transition point of the jamming transition.

In order to study the density fluctuations, we make the time series of the density measured at a local section in the closed circuit. We take the length of the circuit as $L=100000$ and the length of the local section for the observations as 20 . The PSD of the time series is calculated by the Fourier transformations. The result for $\rho=0.19$, which is in the free flow phase, is shown in Fig. 2. Clear power law behavior like $1 / f^{\alpha}$ on the frequency $f$ is seen in the PSD. We find that $\alpha \sim 1.8$.

The PSD in the congested flow phase for $\rho=0.20$ is shown in Fig. 3. In contrast with the free flow phase, no power-law behavior is seen; white-noise-like behavior is observed instead, within a wide range of the frequency. To see more clearly the difference in the PSD in these two phases, we show two PSD's, both for the concentrations much closer to $\rho_{c}$, in Fig. 4: one for the concentration just below the transition, $\rho=0.197575$, and another for the concentration just above the transition, $\rho=0.197576$. The figure shows that the power-law behavior is really seen only below the transition and disappears above the transition; in other words, long-time correlations in the traffic current fluctuation persist in the free flow phase and are destroyed by the occurence of the jamming transition.

To study the origin of the power-low fluctuation in the free flow phase, we make a snapshot of the density profile for $\rho=0.19$ and $L=100000$, which is shown in Fig. 5. The density profile is calculated by dividing the system into 1000 segments. Thus the flucutuations in the scale shorter than 100 are averaged out. In this scale, a number of large clusters of the vehicles are seen in the density profile. Clearly, each cluster is lead by the slowest vehicle within it, that is, one having the slowest preferred velocity. Other faster vehicles are forced to be stuck behind it. Some clusters show internal density fluctuations and some are non-fluctuating. The average density in the non-fluctuating clusters is found as $\sim 1 / 3$, that is, the average headway distance is $\sim 2$ there (the average head-to-head distance is $\sim 3$ ). The existence of this characteristic headway distance is the origin of the sharp high frequency peak seen in the PDS in Fig. 2. The low frequency cutoff in the PDS, on the other hand, is understood as the inverse of the average time which a vehicle take to travel around the whole system.

We also show the histogram of the measured headway distance $h$ in Fig. 6 . The characteristic headway distance 2 is seen also in this figure. Moreover, the headway distance distribution shows a clear power-law behavior of the form $1 / h^{\beta}$ with $\beta \sim 3.0$ in the range from $h \sim 3$ to $h \sim 70$, which corresponds to the scale within the cluster in Fig. 5. Larger headway distances, on the other hand, correspond to the intercluster scale.

It is clear that in the free flow phase of the present model all the vehicles would eventually form a single large cluster, which is headed by the slowest vehicle among all. We, however, see a number of clusters in Fig. 5. Thus the whole system has not yet reached the final stationary state. That is, the power-law fluctuations found above are in non-stationary states. In fact, it is expected to take a very long time for the stationary state to be achieved, since there are a number of slow vehicles whose preferred velocities are close to each other. At the same time, we have no reason as well to expect that the stationary state is achieved in the real traffic flow. Therefore, we think that the power-law fluctuations found in the simulations are actually related to the one observed in the real traffic flow.

To summarize, we found $1 / f^{\alpha}$ type behavior in the PSD of the temporal density fluctuations in the free flow phase of the CML model. The model follows a deterministic dynamics; that is, it develops deterministically into a state which shows the power-law fluctuation. At the same time, the vehicles form the clusters, within which the headway distance distribute with the power-law, $\sim 1 / h^{3.0}$. This power 
law distribution is considered as the origin of the $1 / f^{\alpha}$ fluctuation. In the congested flow phase, on the other hand, the power law fluctuation is not seen; in other words, the occurence of the traffic jam destroys long time correlations in the traffic current fluctuation. It is in contrast with the result by Takayasu and Takayasu, 3] in which the power law was observed only in the congested phase, and also with the one by Nagel and Paczuski [4], who found the power law only near the transition point. The reason why the long-time correlations are destroyed by the traffic jam in the present model is understood as follows: Once a vehicle runs into a jam region, it is forced to stop. When running out of the jam region, it starts again from the beginning so that the memory of the current fluctuations is lost then. We obtained $\alpha \sim 1.8$, the power which is somewhat larger than the values obtained from the real

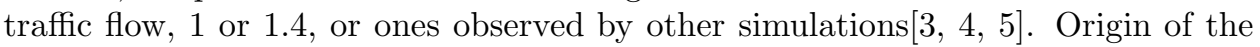
difference in the values of $\alpha$ is not yet clear. Other sort of power-law phenomena than the $1 / f^{\alpha}$ current fluctuation were also reported by some simulations 8 , 10, 过. To clarify their relations is also a problem to be left for future studies.

A subject related to the density fluctuations in the traffic flow is the $1 / f^{\alpha}$ fluctuations in granular flows: By experiments of a narrow channel like a pipe or a hourglass, the power-law fluctuations like $\alpha=1$ or $\alpha=1.5$ were observed 11, 12]. Computer simulations were made recently to reproduce these power law. 113, 14, 15, 16]. It would be worth pointed out that the real experiments of granular flows are made in non-stationary states of the flow, as in the case of the present study. The traffic flow and the granular flow share several similar properties, and have many different points as well. To make their relations clear is a challenging problem. Especially, we found the power-law distribution of the headway distance, which we think is the origin of the $1 / f^{\alpha}$ fluctuation. It would be interesting to study corresponding quantity in the granular flow.

We are grateful to Y. Akutsu, K. Tokita, and T. Nagao for valuable discussions. We also thank S. Tadaki, M. Bando, K. Hasebe, M. Kawahara, H. Ozaki, N. Ito, M. Takayasu and H. Takayasu for valuable discussions. We are grateful to H. Herrmann

for sending us of ref. [8]. Numerical calculation were done mainly by Fujitsu VPP 500 in the Institute for Solid State Physics, the University of Tokyo and NEC SX$3 / 14 \mathrm{R}$ in the computer center of Osaka University.

\section{References}

[1] T. Musha and H. Higuchi: Japan. J. Appl. Phys. 15 (1976) 1271.

[2] T. Musha and H. Higuchi: Japan. J. Appl. Phys. 17 (1978) 811.

[3] M. Takayasu and H. Takayasu: Fractals 1 (1993) 860.

[4] K. Nagel and M. Paczuski: Phys. Rev. E51 (1995) 2909.

[5] X. Zhang and G. Hu: Phys. Rev. E52 (1995) 4664.

[6] S. Yukawa and M. Kikuchi: J. Phys. Soc. Jpn. 64 (1995) 35.

[7] Theory and Application of Coupled Map Lattices, ed. K. Kaneko (Wiley, New York, 1993) and references therein.

[8] K. Nagel and H. Herrmann: Physica A 199 (1993) 254.

[9] K. Nagel and M. Schreckenberg: J. Phys. I France 2 (1992) 2221.

[10] M. Leibig: Phys. Rev. E49 (1994) 184.

[11] K. L. Schick and A. A. Verveen: Nature 251 (1974) 599. 
[12] S. Horikawa, A. Nakahara, T. Nakayama, and M. Matsushita: J. Phys. Soc. Jpn. 64 (1995) 1870.

[13] G. Peng and H. J. Herrmann: Phys. Rev. E49 (1994) R1796.

[14] T. Pöschel: J. Phys. I France 4 (1994) 499.

[15] G. H. Ristrow and H. J. Herrmann: Phys. Rev. E50 (1994) R5.

[16] G. Peng and H. J. Herrmann: Phys. Rev. E51 (1995) 1745.

\section{Figure Captions}

Fig. 1 Plot of the flow $q$ against the concentration $\rho$ measured for the whole system. The length of the system is 1000 . Average over 1000 steps are taken after 5000 steps being discarded. The average over 10 samples are shown.

Fig. 2 The log-log plot of the power spectral density of the temporal density fluctuation. Concentration of vehicle is $\rho=0.19$. The system length $L$ and a length of observation section are 100000 and 20, respectively. We record a time series of 65536 steps after discarding 400000 steps. The average over 30 samples are shown. The slope of the straight line is -1.8 .

Fig. 3 The log-log plot of the power spectral density of the temporal density fluctuation for $\rho=0.20$. The length parameters and the simulation steps are same as Fig. 2. The result of a single sample is shown.

Fig. 4 The log-log plot of the power spectral densities. The system length is $L=$ 200000 and the length of local section for observation is 20 . We record the time series for 32768 steps after discarding 800000 steps. No sample average is taken. (a) $\rho=0.197575$, which is just below the transition. (b) $\rho=0.197576$, which is just above the transition.

Fig. 5 Snapshot of the density profile for $L=100000$ and $\rho=0.19$. We take it after discarding 500000 time steps. Density is calculated by dividing the system into 1000 segments.

Fig. 6 The log-log plot of the distribution histogram of the headway distance for $L=100000$ and $\rho=0.19$. Distribution is calculated in 100 steps after discarding 500000 steps. The slope of the straight line is -3.0 . 